\title{
RESPONSABILIDAD DE ROL Y DIRECTRICES
}

Pablo Larrañaga

Universidad de Alicante

\section{Introducción}

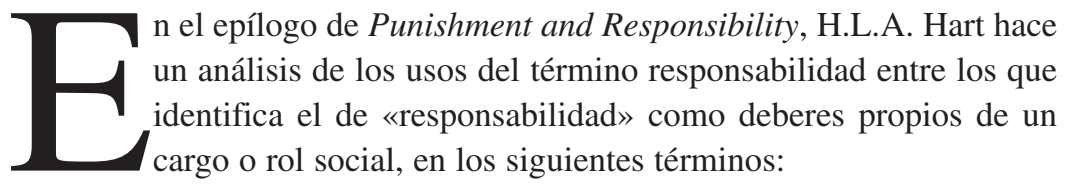

«[... siempre que una persona ocupe un lugar o puesto determinado en una organización social, respecto del cual se asignen deberes específicos para promover el bienestar, o para impulsar de manera específica las metas o propósitos de la organización, se dice con corrección que esta persona es responsable del cumplimiento de estos deberes o de hacer lo necesario para satisfacerlos» ${ }^{1}$.

El mismo Hart apunta que esta generalización no puede llevarse muy lejos sin que surjan dudas acerca de los casos marginales. Como consecuencia de ello, al tratarse de un criterio considerablemente vago, puede sernos útil para identificar ciertos deberes específicos en determinados casos centrales, pero deja sin aclarar casos periféricos en los que, por ejemplo, las posiciones sociales no sean fáciles de determinar o cuando, respecto de un mismo rol, concurran expectativas de conducta incompatibles. Este tipo de dificultades se enfrentan, creo, en el caso de no pocos roles sociales; al menos, en el de muchos singularmente importantes en las sociedades contemporáneas, relacionados, por ejemplo, con la atribución de responsabilidades dentro de grandes organizaciones como el gobierno y las empresas. Por este motivo, me parece oportuno detenerse en una consideración más profunda de la idea apuntada por Hart.

Este trabajo parte de la permisa de que la mejor manera de aproximarse al concepto de responsabilidad es teniendo en cuenta que se trata de un concepto normativo; en el sentido de que su definición depende de la referen-

\footnotetext{
${ }^{1}$ En Hart, H.L.A., «Postscript: Responsibility and Retribution», Punishment and Responsibility, Clarendon Press, Oxford, 1988, p. 212. Cursivas mías.
} 
cia a las normas (jurídicas, morales, políticas) de las que, explícita o implícitamente, depende el uso típico del término. En otra ocasión he tratado de formular una definición del concepto de responsabilidad esforzándome en poner de manifiesto el entramado normativo al que se refiere este uso del término «responsabilidad» en el ámbito jurídico² ${ }^{2}$ En este trabajo, utilizando como punto de partida una reformulación de esa definición, trato de precisar con mayor detalle los distintos tipos de normas, de hechos y de relaciones normativas que abarca este concepto. Esta nueva aproximación a la definición tiene por objeto, en primer lugar, mostrar la especificidad de las obligaciones vinculadas con roles que solemos calificar como «responsabilidades» $y$, en segundo lugar, señalar el tipo de normas que, a mi juicio, utilizamos para disciplinar este tipo de deberes especiales.

Este trabajo se divide en cuatro partes. En la primera, se postula una definición del concepto de responsabilidad como deberes de un rol o cargo, y se acota el subsiguiente análisis a una de las condiciones de justificación y a una de las funciones que realiza el término «responsabilidad» en las frases en las que, en el sentido que estamos tratando, se utiliza en su forma típica. En la segunda parte, me detengo a examinar la especificidad de los deberes especiales que calificamos como responsabilidades, señalando los rasgos que, desde mi punto de vista, distinguen este tipo de deberes de otros deberes especiales a los que no solemos calificar como responsabilidades. En la tercera parte, a partir de la consideración de sus rasgos específicos, me pregunto qué tipo de normas resulta más adecuado para dar cuenta de las responsabilidades de rol. Por último, en la cuarta parte, me detengo en algunas consideraciones respecto de la relación entre la teoría de los enunciados jurídicos y la teoría de la responsabilidad.

Como se verá, el análisis anterior conduce a tres conclusiones. En primer lugar, que los deberes especiales vinculados con roles o cargos y que solemos llamar «responsabilidades» se caracterizan, por un lado, porque están relacionados con la consecución de objetivos y, por otro, porque tienen un componente de deliberación ajeno a otros tipos de deberes. En segundo lugar, que entre los distintos tipos de normas de mandato, las directrices son las que mejor sirven para dar cuenta de los deberes especiales consistentes en alcanzar objetivos y que dependen de la deliberación por parte del sujeto respecto de la conducta específica efectivamente prescrita; esto es, que las directrices son el mejor candidato para constituir la base normativa de las responsabilidades de rol. En tercer lugar, como consecuencia de poner en relación la teoría de los enunciados jurídicos con la teoría de la responsabilidad, una tercera conclusión -que podemos considerar paralela al tema

\footnotetext{
${ }^{2}$ Larrañaga, Pablo, El concepto de responsabilidad, Fontamara, México, 2000, pp. 155 ss.
} 
del trabajo- lleva a plantear la necesidad de elaborar con mayor detalle la categorización de las directrices como mandatos de optimización, en particular, en lo que se refiere a la manera en la que se configura su medida de cumplimiento. En este sentido, para que la categoría de las directrices sea compatible con la teoría de la responsabilidad, es necesario mostrar cómo se determinan los estándares de conducta en virtud de los cuales, sobre la base de este tipo de normas, se formulan juicios de responsabilidad.

\section{La definición de responsabilidad como deberes propios de un rol o cargo y el planteamiento del problema}

Siguiendo el método de definición o elucidación de los conceptos jurídicos propuesto por Hart, consistente en señalar las condiciones para que sean verdaderas las frases en las que un término jurídico tiene su función típica y cómo se usan para extraer conclusiones de las normas en casos particulares $^{3}$, se puede postular la siguiente definición de responsabilidad como deberes propios de un cargo o rol social:

1) Un enunciado de la forma «Dado que $X$ tiene el cargo $C$, $X$ es responsable de $\mathrm{Y} »$ es verdadero cuando se satisfacen las siguientes condiciones:

a) existe un sistema normativo ( $\mathrm{SN}$ );

b) en el sistema normativo ( $\mathrm{SN}$ ) existe una institución (I); una regla o un conjunto de reglas constitutivas (RC) mediante la(s) cual(es) X constituye una instancia de la institución (I) [o es un caso individual de la institución (I)], y un conjunto de normas regulativas (RR) entre las que existe, al menos, una norma (D) que indica uno o varios objetivos (Y) que debe alcanzar la institución (I);

c) ocurre, de hecho, que $X$ es un caso particular de la institución (I);

d) ocurre, de hecho, que $\mathrm{Z}$ es una conducta o curso de acción que tiene una relación causal (medio-fin) con el (los) objetivo(s) «Y», establecido(s) por la norma (D).

2) Los enunciados del tipo «Dado que $X$ tiene el cargo $C$, es responsable de Y» se utilizan:

a) para especificar deberes especiales de $\mathrm{X}$ (relativos a la conducta $\mathrm{Z}$ ) $\mathrm{y}$,

b) para obtener conclusiones normativas que pueden servir, eventualmente, como antecedentes a juicios en los que el término «responsabilidad» opere en el sentido de sancionabilidad ${ }^{4}$.

\footnotetext{
${ }^{3}$ Creo que en esta ocasión no es necesario reproducir la formulación de dicho método in extenso, ni mostrar su utilidad para el análisis de algunos conceptos normativos. Sin embargo, al respecto puede verse Hart, H.L.A., «Definición y teoría en la ciencia jurídica», en Derecho y Moral. Contribuciones a su análisis, (trad. Genaro Carrió), Ed. Depalma, Buenos Aires, 1962, pp. 93-138 y, relacionado en concreto con el concepto de responsabilidad, Larrañaga, op. cit., El concepto de responsabilidad, pp. 85-98.

${ }^{4}$ Para un análisis más amplio de esta definición y, en particular, de la relación entre el uso de «responsabilidad» como deberes propios de un rol o cargo y el de «responsabilidad» como «sancionabilidad», cfr. Hart, op. cit., «Postscript: Responsibility and Retribution», Punishment and Responsibility, passim; y Larrañaga, op. cit., El concepto de responsabilidad, pp. 151-189 y 194-196.
} 
Atendiendo a los objetivos señalados anteriormente, en este trabajo me ocuparé fundamentalmente de la relación entre las condiciones enumeradas en el punto (1)-en particular, de la condición (d) - y la función (a) de dichos enunciados. En otras palabras, dejando a un lado el problema de quién es responsable -i.e. quién y con qué justificación ocupa un rol o cargo-, este trabajo gira en torno a cuáles son sus responsabilidades. Esto es, se dirige al problema de cómo se determina el contenido de expectativas de conducta del rol o, en términos de la definición anterior, cómo determinamos el deber específico de hacer u omitir la conducta Z. En este sentido, el tipo de problemas que tengo en mente son los relativos a casos como los siguientes: si, como padre de familia, $\mathrm{X}$ es responsable del bienestar de sus hijos, ¿qué es exactamente lo que tiene que hacer u omitir? O bien, si la salud pública entra dentro de las competencias del Ministerio de Sanidad, ¿qué tiene que hacer u omitir el ministro, o la ministra, correspondiente para evitar que se extienda una epidemia?, ¿o para que la gente fume menos?, ¿o para que, en general, cumpla con su responsabilidad de que la gente esté más sana?

Ahora bien, creo que antes de entrar de lleno en la materia resulta oportuno hacer algunas aclaraciones respecto de la anterior definición.

En primer lugar, una cuestión terminológica que, en mi opinión, resulta relevante para encuadrar la naturaleza del problema. En su artículo «El enunciado de responsabilidad» ${ }^{5}$, Ernesto Garzón Valdés llama «enunciados de responsabilidad prospectivos» a los enunciados que realizan la función de adscripción de deberes especiales reconocidos como «responsabilidades», mientras que cuando cumplen su función dentro de los juicios de responsabilidad como sancionabilidad, se refiere a ellos como «enunciados de responsabilidad retrospectivos». En este sentido, se diría que el objeto central de este trabajo son los enunciados de responsabilidad prospectivos vinculados con roles. No obstante, en adelante utilizaré la expresión «juicio de responsabilidad prospectivo». Mi preferencia se basa en que, en mi opinión, tomando en cuenta la naturaleza normativa del concepto a la que me he referido antes, el término «juicio» denota mejor que el término «enunciado» la función adscriptiva o imputativa y la dimensión de derrotabilidad con las que normalmente vinculamos este concepto. La idea de responsabilidad es adscriptiva porque, como se desprende de la anterior definición, el sentido de enunciados del tipo «X es responsable de $\mathrm{Y}$ » depende de la aplicación de un conjunto sistemático de normas constitutivas y regulativas; de modo que dicho enunciado tiene, fundamentalmente, un carácter directivo-valorativo,

\footnotetext{
${ }^{5}$ Garzón Valdés, Ernesto, «El enunciando de responsabilidad», Doxa. Cuadernos de Filosofía del Derecho, 19, Alicante, 1996, p. 260.
} 
consecuencia de la aplicación de dicho conjunto de normas y no, como parecería indicar el término «enunciado», una función descriptiva dependiente de la posesión de ciertas características o propiedades ${ }^{6}$. Y es derrotable, porque tal adscripción tiene un carácter prima facie ya que, en principio, negando que se haya producido cualquiera de las condiciones de validez o de justificación señaladas en la definición, puede cancelarse la función del enunciado ${ }^{7}$. Así pues, tomando esto en cuenta, podría decirse que me ocupo de las condiciones de validez o justificación de los juicios de responsabilidad prospectivos vinculados con roles.

En segundo lugar, para los objetivos que he planteado resulta, en principio, indiferente que la condición (a) de la definición - esto es, el sistema normativo de referencia- sea algún tipo de sistema normativo en concreto (jurídico, moral, político, etc.). En el nivel de abstracción en el que me propongo desarrollar esta aproximación a las responsabilidades vinculadas con roles, no es necesario hacer referencia a una clase específica de normas; así que la responsabilidad en cuestión podría adoptar el carácter del sistema normativo al que se haga referencia ${ }^{8}$. No obstante, en la medida en la que para la descripción de los elementos de la definición cuento con una teoría

\footnotetext{
${ }^{6}$ En este aspecto sigo la idea alguna vez sostenida por Hart de que la función de enunciados del tipo en los que se utiliza el término «responsabilidad» es, fundamentalmente, adscriptiva y no descriptiva. En este sentido, aunque el mismo Hart haya rechazado al adscriptivismo en su conjunto como teoría de la acción humana ( $c f r$. Hart, op. cit., Punishment and Responsibility, prefacio) creo que sigue siendo acertada su intuición fundamental respecto de cómo usamos ciertos términos entre los que se encuentra el de «responsabilidad». Al respecto, $c f r$., Hart, H.L.A., «The Ascription of Responsibility and Rights», Proceedings of the Aristotelian Society, 49, 1948-1949, pp. 171-194. Para una perspectiva crítica, sobre todo relativa al adscriptivismo como teoría de la acción, $c f r$, por ejemplo, Backer, G.P., «Defeasibility and Meaning», en Hacker, P.S., y Raz, J. (eds.), Law, Morality and Society. Essays in Honour of H.L.A. Hart, Clarendon Press, Oxford, 1992, pp. 26-57; Geach, P.T., «Ascriptivism», Philosophical Review, 1960, pp. 221- 225 y Pitcher, G., «Hart on Action and Responsibility», Philosophical Review, 1960, pp. 226-235. Para una muestra de utilidad de las nociones de derrotabilidad o revocabilidad en el análisis de la responsabilidad, cfr., por ejemplo, Feinberg, J., «Acción y responsabilidad», en White, A. (ed.), La filosofía de la acción, FCE, México, 1976, pp. 139-174. Respecto de la relación entre el carácter adscriptivo y la dimensión de derrotabilidad asociada a la noción de responsabilidad, cfr. Larrañaga, op. cit, El concepto de responsabilidad, pp. 196 ss.

${ }^{7}$ El elemento de la derrotabilidad se relaciona, obviamente, con el complejo problema de las justificaciones y las excusas. En este sentido, las condiciones de validez de los juicios de responsabilidad no dependen sólo de los elementos de cada una de las definiciones, sino que deben vincularse con los principios generales que disciplinan la imputación a los distintos ámbitos normativos. Además, en el caso de la responsabilidad de rol, la cuestión de la derrotabilidad respecto de juicios en los que se especifica una conducta particular puede complicarse considerablemente a causa de la potencial complejidad de la relación medio-fin, entre las conductas debidas y el objetivo respecto del cual se dice que alguien es responsable -i.e. de lo que llamo razones de oportunidad y razones de eficiencia-.

${ }^{8}$ Larrañaga, op. cit., El concepto de responsabilidad, pp. 18 ss.
} 
de los enunciados jurídicos más desarrollada que sus equivalentes en otras áreas, utilizaré las categorías desarrolladas dentro de la teoría de Derecho.

En tercer lugar, aunque en la formulación de las normas de conducta que disciplinan la responsabilidad de rol no se suela aludir directamente a las conductas, sino sólo a objetivos (estados de cosas) y se deje, por lo general, para un momento ulterior - de la aplicación de la norma o de la formulación de juicios retrospectivos- la especificación de la conducta efectivamente debida - esto es, el contenido de la norma sólo se determina mediante un proceso de deliberación en un contexto de imputación específico-, tanto en la condición (d) como en la función a) me refiero a «Z» como una conducta con una relación causal, o medio-fin, con el objetivo prescrito por una norma. Esta explicitud responde a que la justificación de juicios de responsabilidad depende de la especificación de los estándares de conducta efectivamente exigibles que, a su vez, dependen de la individualización de acciones. Por ello, desde mi punto de vista, en una definición general conviene explicitar directamente este elemento.

Por último, en cuarto lugar, cuando me refiero a una relación causal o relación medio-fin, tengo en mente la amplia noción de causalidad que suele utilizarse en contextos adscriptivos o imputativos, según la cual, por un lado, «causar» equivale a intervenir en el estado del mundo existente o esperable y, por otro, tales intervenciones pueden consistir, además de en acciones naturales, en relaciones convencionales, en motivaciones interpersonales y en oportunidades ${ }^{9}$.

Lo que quiero enfatizar utilizando la noción de causalidad es que, en mi opinión, el contexto de la responsabilidad de rol es el de las relaciones extrínsecas -no de las analíticas ni de las convencionales- entre las acciones o conductas y las consecuencias de éstas. Esto es, las responsabilidades se refieren a cambios o modificaciones de estados de cosas cuyas propiedades relevantes para la descripción son distintas de las que utilizamos para individualizar la acción -i.e. del resultado natural o institucional de que se trate-. Desde mi punto de vista, el tener en consideración este hecho es lo que permite que, por ejemplo, con independencia de la naturaleza del concepto de justicia que adoptemos, podamos distinguir entre el deber del juez de ser justo (o, para simplificar, el deber de dictar sentencias conforme a Derecho) y la responsabilidad del juez de que se haga justicia. Esto es así porque, mientras que la acción que satisface dicho deber puede describirse en tér-

${ }^{9}$ Cfr., Hart, H.L.A. y Honoré, Tony, Causation in the Law, Clarendon Press, Oxford, 1985, cap. II, passim. Y para un desarrollo más amplio de la teoría, aplicada específicamente a la problemática de la responsabilidad, cfr. Honoré, Tony, Responsibility and Fault, Hart Publishing Co., Oxford, 1999, pp. 94 ss. 
minos estrictamente analíticos o convencionales (formales), definitorios de la acción institucional contenido del deber, determinar la conducta que satisface la responsabilidad es una cuestión que exige aludir a cambios en el mundo que sólo pueden ser consecuencia de acciones, pero no meramente su(s) resultado(s). Así pues, desde este punto de vista, sólo podemos entender lo que significa «hacer» justicia -y, por tanto, obviamente, la responsabilidad de que se haga justicia- si tenemos en cuenta las consecuencias en el mundo de las acciones naturales y/o convencionales (institucionales) del juez en términos, por ejemplo, de modificaciones de las relaciones sociales existentes. Esto no sería posible si nos limitáramos a analizar los cambios analítica o normativamente necesarios para considerar que la acción de que se trate ha tenido lugar - por ejemplo, dictar una sentencia conforme a Derecho-. Es decir, cuando, por definición, dado un conjunto determinado de normas, se ha dictado una sentencia «justa».

Pero, ¿cómo distinguimos, entonces, el deber especial de imparcialidad de los jueces de su responsabilidad de que se haga justicia?; ¿dónde reside la diferencia entre la obligación de los padres de suministrar alimentos a sus hijos y la responsabilidad de promover el bienestar de éstos?; ¿por qué el médico, responsable de procurar la curación de sus pacientes, no cumple con sus responsabilidades por el mero hecho de no infringir los deberes de la práctica clínica? A este tipo de preguntas trataré de responder en las próximas páginas.

\section{Deberes y responsabilidades}

Este trabajo parte del postulado de que, en su función prospectiva, tener «responsabilidad» no puede sustituirse por tener un «deber», sin alterar el significado usual del término ${ }^{10}$. Hart se plantea esta cuestión de la siguiente manera:

\footnotetext{
${ }^{10}$ Esta no es, sin embargo, una opinión unánime. Al respecto, por ejemplo, Garzón Valdés afirma lo siguiente: «En los ERs [enunciados de responsabilidad, P.L.] prospectivos lo que se afirma es que alguien tiene la responsabilidad de procurar que se dé algún estado de cosas futuro. Por ejemplo, cuando se dice que Pedro es responsable del cuidado del jardín de Juan durante las vacaciones de este último, es decir, que Pedro es responsable de que las flores del jardín de Juan no se marchiten por falta de riego. La expresión 'es responsable' puede ser remplazada sin mayor alteración de significado por la expresión 'tiene el deber o la obligación'»; Garzón, op. cit, «El enunciado de responsabilidad», p. 260. Cursivas mías. Por su parte, Michael J. Zimmerman considera que en los enunciados de responsabilidad prospectivos «tener una responsabilidad» (to bear a responsibility) puede sustituirse, sin más, por «tener una obligación o un deber»; «Responsibility», Encyclopaedia of Ethics, Becker, L. C. y Becker, C.B., (eds.), Garland, Nueva York, 1992, p.1089. Aunque, evidentemente, la interpretación que se haga de «sin mayor alteración de significado» seguramente nos llevaría, en muchos casos, a un acuerdo respecto de una sinonimia, a diferencia de Garzón Valdés y de Zimmerman considero, por un lado, que una parte central del análisis del concepto de responsabilidad en relación
} 
«[...] es importante tener en cuenta que no todos los deberes que alguien tiene en virtud de ocupar lo que en un sentido estricto sería un «rol» se consideran o se llaman «responsabilidades». Un soldado tiene la obligación de obedecer a su oficial superior, y si éste le ordena cuadrarse o presentar armas en una ocasión determinada, tiene el deber de hacerlo. Pero difícilmente se diría que cuadrarse o presentar armas es su responsabilidad; tampoco se diría que es responsable de hacer esto. Pero si, por otro lado, al soldado se le ordenara llevar un mensaje al cuartel general o llevar prisioneros hasta el campamento base, bien podría decirse que él es responsable de hacer tales cosas y que tales cosas son sus responsabilidades. Creo, aunque confieso que no estoy seguro de ello, que lo que distingue estos deberes de rol que son señalados como responsabilidades es que se trata de deberes de un tipo relativamente complejo y amplio que definen una «esfera de responsabilidades» que requiere cuidado y atención a lo largo de un prolongado período de tiempo, mientras que los deberes de corta duración o de tipo simple, relativos a hacer o no hacer algo en una ocasión particular, no son llamados responsabilidades. Así, el soldado a quien se le asigna mantener limpio el campamento en vistas a la visita de inspección del general, tiene esta esfera de responsabilidades. Pero si simplemente se le indica que recoja un papel del suelo al aproximarse el general, este sería, en todo caso, su deber» ${ }^{11}$.

Desde mi punto de vista, aunque al caracterizar las «tareas» asignadas al soldado Hart intuye el núcleo de la distinción entre deberes y responsabilidades, yerra al momento de especificar los criterios de la misma. Así pues, aunque probablemente en la mayoría de los casos la complejidad y duración de los deberes aportan algunas claves importantes para la distinción, creo que en otras circunstancias resultan criterios insuficientes.

Por un lado, podemos encontrar deberes simples y de corta duración a los que llamaríamos «responsabilidades». Imaginemos que Alicia y Bernardo deciden ir a pasar un par días al campo, que le piden a Carolina que vaya a su casa para dar de comer a su gato o para regar las plantas, y que ella acepta hacerles ese favor. Creo que en estas circunstancias puede decirse con plena corrección que Carolina ha adquirido la responsabilidad de darle de comer al gato o de regar las plantas el día o los días que hayan acordado. El deber es simple, en el sentido de que sólo requiere una acción o pocas acciones específicas; y es de corta duración, pues se agota en el momento en que le ha dado de comer al gato o ha regado las plantas, a lo sumo, una o dos veces.

Por otro lado, podemos encontrar deberes complejos que se prolongan en el tiempo, y que no llamaríamos responsabilidades. En este caso, pode-

con los roles consiste, precisamente, en establecer los contextos en que se puede distinguir entre tener una responsabilidad y tener una obligación o deber especial tout court y, por otro, que esa diferencia tiene relevancia teórica.

${ }^{11}$ Hart, op. cit. Punishment and Responsibility, p. 213. Cursivas mías. 
mos pensar en el deber de respeto mutuo entre los cónyuges. Se trata de un deber complejo, en el sentido de que múltiples acciones y omisiones pueden ser significativas de respeto o bien constituir faltas de respeto, dependiendo de las circunstancias. También se trata, en ocasiones, de un deber que se prolonga durante largo tiempo; años, incluso décadas.

Pero, si la complejidad y la permanencia no son criterios adecuados, ¿cómo damos cuenta entonces de la distinción de la que quiere dar cuenta Hart?

En mi opinión, la línea divisoria entre deberes y responsabilidades tiene que ver con dos aspectos que caracterizan a los deberes especiales que llamamos «responsabilidades», ajenos a deberes de otro tipo: por un lado, se trata de deberes que se vinculan con la consecución de objetivos y, por otro, incluyen un ingrediente de deliberación o de discrecionalidad respecto de la conducta efectivamente prescrita ${ }^{12}$.

En esta línea de ideas, la razón por la que calificamos como «responsabilidad» el deber simple y breve de dar de comer al gato o de reglar las plantas radica en que, por un lado, su contenido se asocia con objetivos -que el gato esté alimentado o que las plantas no se sequen-y, por otro, estos objetivos se pueden alcanzar mediante los cursos de acción que se consideren adecuados dadas las circunstancias, y respecto de cuya idoneidad debe deliberar el sujeto responsable -Carolina puede, por ejemplo, pedirle a otra persona que riegue en su lugar; puede instalar un comedero automático para que el gato coma cuando le apetezca; puede ponerle mucha comida de una vez, o puede darle comida en pequeñas raciones, poco a poco, etc.-. Por otra parte, la razón por la que no llamamos «responsabilidades» al complejo y duradero deber de respeto mutuo entre los cónyuges es porque este deber no está asociado directamente con ningún objetivo particular, ni tampoco requiere, para determinar la conducta exigida, de una deliberación circunstancial por parte de los sujetos. Para guiar su conducta, los cónyuges no tienen por qué tener en cuenta ni las consecuencias del respeto ni las de la falta de respeto; simplemente tienen que evitar cualquier conducta o actitud que, en cualquier momento y en cualquier forma, pudiera calificarse de ese modo.

\footnotetext{
${ }^{12}$ Antes de continuar con el análisis conviene hacer una estipulación terminológica: en lo que sigue hablaré de «resultado» para referirme a un estado de cosas que es consecuencia de una acción natural o institucional o, por decirlo de algún modo, a los cambios en los estados de cosas. Y utilizaré «objetivo» cuando aluda un estado de cosas cuya obtención viene prescrita por una norma. En este sentido, en los términos de este trabajo, los «objetivos» son estados de cosas cualificados por una norma de conducta. Esta cualificación no es sólo deóntica necesariamente, sino que puede incluir algunas cualidades o propiedades del estado de cosas en cuestión o de su modo de producción.
} 
Así pues, teniendo en cuenta lo anterior, cuando en el ejemplo de Hart se le asigna al soldado la tarea de limpiar el campamento se hacen dos cosas distintas. En primer lugar, se señala un objetivo que debe alcanzar: que el campamento esté limpio. Debe alcanzar este objetivo a lo largo de un proceso más o menos complejo que supone múltiples acciones -como barrer, limpiar ventanas, recoger basura, etc.- cuya realización no tiene individualmente una relación intrínseca con el objetivo buscado, sino que cada una va completando el contexto causal. En segundo lugar, lo que es más importante, aunque se le señala el objetivo que debe perseguir, ni se le indica qué acciones concretas debe realizar ni se le señala en qué orden concreto debe llevar a cabo el conjunto de cursos de acción vinculado causalmente con aquél. El asunto de determinar qué hacer y cómo hacerlo depende de él: decimos que queda bajo su responsabilidad. Para realizar su tarea, el soldado tendrá que realizar múltiples acciones, desde barrer hasta limpiar letrinas, pasando por bruñir bronces, etc., cuyo conjunto agregado, en el mejor de los casos, supondrá que se alcance el objetivo señalado. En otras palabras, el objetivo que se busca al asignarle al soldado, la «tarea» que se le encomienda es, obviamente, que el campamento llegue a estar limpio; pero las acciones conducentes a ello y cómo hacer compatible este objetivo con otras exigencias de la vida castrense, son asuntos que dependen de su discreción; quedan bajo su responsabilidad.

Tomando en cuenta lo anterior, parece que la idea de Hart de que las acciones debidas que llamamos responsabilidades componen una «esfera de responsabilidades» -en alguna medida compleja y con cierta tendencia a perdurar-, aunque imprecisa, tiene pleno sentido en la medida que refleja la complejidad de las relaciones medios-fines que subyacen a la asignación de tareas. En este contexto, las responsabilidades no se refieren a conductas específicas, sino a un conjunto de acciones indeterminadas o de cursos de acción indeterminados -aunque no indeterminables-, que corresponde realizar a la persona a la cual se le asigna una tarea. De allí que el término «responsabilidad» en el sentido que venimos tratando pueda referirse a tres cosas distintas, pero íntimamente relacionadas entre sí: 1) al objetivo asignado (el soldado es responsable de que el campamento esté limpio); 2) a la actividad o curso de acción conducente a alcanzar un objetivo (el soldado es responsable de limpiar) y 3) a las acciones específicas que componen la actividad conducente a alcanzar un objetivo (el soldado es responsable, por ejemplo, de barrer $)^{13}$. En cualquier caso, en mi opinión, el carácter complejo y pro-

\footnotetext{
${ }^{13}$ Así pues, retomando los términos de la definición anterior, en virtud de la orden de mantener limpio el cuartel, podemos decir que el lugar o puesto que le corresponde a este soldado dentro de la organización social es el de «soldado de la limpieza». Decimos, entonces, que es
} 
longado de los deberes que componen la esfera de responsabilidades a la que alude Hart puede verse como la combinación de la asignación de una tarea y la discrecionalidad en la realización de ésta. En este sentido, por un lado, los conjuntos de posibles acciones respecto de cuya idoneidad debe deliberar el sujeto responsable de realizar una tarea suelen ser complejos y, por otro, los objetivos que requieren la asignación específica de una tarea a una persona suelen ser producto de cursos de acción desarrollados en un período de tiempo y no de un acto único.

Ahora bien, si, como he afirmado, la responsabilidad de rol se caracteriza, por un lado, por consistir en deberes vinculados con la consecución de objetivos y, por otro, porque los sujetos tienen que deliberar acerca de la relación causal entre sus acciones y tales objetivos, ¿cómo damos cuenta de la existencia de este tipo de deberes? O, dicho en otras palabras, ¿cómo se fundamentan los juicios de responsabilidad prospectivos vinculados con roles?

\section{Responsabilidades, reglas de fin y directrices}

Desde una perspectiva normativa, la importancia que le he dado a los objetivos de los roles y a la discrecionalidad de los sujetos para elegir los medios para alcanzarlos lleva a preguntarse acerca de qué tipo de normas establecen tales objetivos y, consecuentemente, configuran tales marcos de deliberación.

Creo que un buen punto de partida para responder a las preguntas anteriores se encuentra en la teoría de los enunciados jurídicos de Manuel Atienza y Juan Ruiz Manero ${ }^{14}$. Dentro de la categoría de las normas de mandato, estos autores proponen dos categorías que parecen buenos candidatos para responder a las anteriores preguntas: las reglas de fin y las directrices. Las reglas de fin son reglas que prescriben la obtención de estados de cosas de modo que, si se dan determinadas circunstancias, un sujeto debe alcanzar un determinado objetivo, dejando dentro de la discreción del sujeto la elec-

responsabilidad del soldado de la limpieza que el campamento esté limpio: en términos de la definición, «Dado que $\mathrm{X}$-el soldado- tiene el cargo C -es el soldado de la limpieza-, X es responsable de Y -de que el campamento esté limpio-». O decimos, simplemente, que el soldado es responsable de la limpieza del campamento: en términos de la definición, «X -el soldado- es responsable de Y -de que el campamento esté limpio-». Y, también, que el soldado es responsable de realizar las distintas acciones que coadyuven a lograr el objetivo: en términos de la definición, «Dado que $\mathrm{Z}$-por ejemplo, barrer- es una conducta o curso de acción que tiene una relación causal con Y -que el campamento esté limpio-, y dado que «X tiene el cargo $\mathrm{C}, \mathrm{X}$ es responsable de $\mathrm{Y} »$, « $\mathrm{X}$ es responsable de $\mathrm{Z}$-de barrer-».

${ }^{14}$ Atienza, Manuel y Ruiz Manero, Juan, Las piezas del Derecho. Teoría de los enunciados jurídicos, Ariel, Barcelona, 1996. En particular, cap. I. 
ción de los medios idóneos para alcanzarlo ${ }^{15}$. Las directrices también prescriben la obtención de objetivos, pero se caracterizan por dos rasgos distintivos, relevantes ambos para nuestro tema y relativos, por un lado, a la configuración de sus condiciones de aplicación y, por otro, a la configuración del modelo de conducta prescrito. Así, en primer lugar, las directrices configuran sus condiciones de aplicación de manera abierta: es decir, la norma no especifica las condiciones de aplicación, sino que éstas se encuentran mediante un proceso de ponderación en el que el sujeto debe tener en cuenta tanto la presencia de otros deberes y objetivos como los medios disponibles y, en segundo lugar, las directrices operan como mandatos de optimización, constituyendo la prescripción como el deber de alcanzar un objetivo en la mayor medida posible, sin especificar las condiciones para establecer tal posibilidad ${ }^{16}$.

\footnotetext{
${ }^{15}$ Así, en términos de estos autores: «Los sistemas jurídicos contienen también reglas que califican deónticamente no una determinada conducta, sino la obtención de un cierto estado de cosas. Proponemos denominar a las reglas de mandato de este último tipo reglas de fin», ibídem, p. 7. Y sobre la relevancia de la distinción entre reglas de acción y reglas de fin, señalan: «la distinción es relevante cuando la disposición que estipula como obligatoria, por ejemplo, la producción de un determinado estado de cosas deja a la discreción del destinatario la selección de los medios causalmente idóneos para producirlo: en este sentido, las reglas de fin dejan a sus destinatarios un margen de discreción que no existe en las reglas de acción», ibídem, p. 8. Y cuando analizan esta distinción en términos de los tipos de razones que proveen ambos tipos de normas, señalan: «Las reglas de fin constituyen también razones perentorias e independientes del contenido para procurar el estado de cosas prescrito. Ello no obstante, presentan diferencias importantes con las reglas de acción en cuanto al modo de guiar la conducta. Las reglas de acción permiten simplificar el proceso de decisión de quien debe comportarse de acuerdo con ellas (el que debe cumplirlas o controlar su cumplimiento): lo único que debe hacer es comprobar si se han dado o no determinadas condiciones para hacer o dejar de hacer una determinada acción, desentendiéndose de las consecuencias, esto es, del proceso causal que va a desencadenar su comportamiento. Sin embargo, las reglas de fin trasladan al destinatario de las normas el control (o la responsabilidad) de (por) las consecuencias de la conducta», ibídem, pp. 12s.

16 En este punto, Atienza y Ruiz Manero coinciden con Robert Alexy, quien establece la distinción entre reglas y principios en los siguientes términos: «El punto decisivo para la distinción entre reglas y principios es que los principios son normas que ordenan que se realice algo en la mayor medida de lo posible, en relación con las posibilidades jurídicas y fácticas. Los principios son, por consiguiente, mandatos de optimización que se caracterizan porque pueden ser cumplidos en diversos grados y porque la medida ordenada de su cumplimiento no sólo depende de las oportunidades fácticas, sino también de las posibilidades jurídicas»; Alexy, Robert, «Sistema jurídico, principios jurídicos y razón práctica», Doxa. Cuadernos de Filosofía del Derecho, 5, Alicante, 1988, p 143. Cursivas mías. Hay que tener en cuenta, sin embargo, que para Atienza y Ruiz Manero sólo las directrices, y no los que ellos llaman «principios en sentido estricto», operan como mandatos de optimización en el sentido de que sean normas que puedan cumplirse en diversos grados. Atienza y Ruiz Manero, op. cit., Las piezas del Derecho, p. 9. Según Atienza y Ruiz Manero, « [...] lo característico de las directrices se halla en que este tipo de pautas configura de forma abierta tanto sus condiciones de aplicación como el modelo de conducta. Además, no ordenan, ni prohíben, ninguna acción, sino la consecución de un objetivo, de un estado de cosas, sin determinar cuáles sean conductas causalmente idóneas pa-
} 
Si se acepta que, como he sostenido, los elementos que permiten distinguir las responsabilidades de otros deberes son tanto la configuración del objetivo como la discrecionalidad respecto de las conductas, ambas categorías parecen adecuadas para sostener juicios de responsabilidad de rol prospectivos. Sin embargo, creo que en la medida en la que las directrices requieren una deliberación no sólo respecto de la relación medios-fines, sino también respecto de las condiciones que configuran la guía de conducta, son mejores candidatos que las reglas de fin para definir los casos paradigmáticos de la responsabilidad como deberes propios de un rol o cargo. En este sentido, creo que, como puede verse en el ejemplo del soldado, la doble vertiente deliberativa de las directrices ilumina el núcleo de la responsabilidad como deberes de un rol o cargo.

Si consideramos que el objetivo de que el campamento esté limpio viene establecido por una regla de fin, entonces el soldado responsable de alcanzar ese objetivo deberá deliberar acerca de qué conducta, o qué secuencia de conductas, es la más idónea en términos causales para alcanzar ese objetivo. Esto es, deberá hacer consideraciones de tipo estratégico para alcanzar el fin prescrito, pero no tendrá por qué preocuparse de nada más. Eso sí, si fracasa, si resulta que el campamento no llega a estar limpio ${ }^{17}$, diremos que no ha cumplido con sus responsabilidades.

Ahora bien, la situación es mucho más compleja -y también más iluminadora- si consideramos que el objetivo no es prescrito por una regla de fin, sino por una directriz. En este caso, el soldado enfrenta dos tipos deliberaciones: por un lado, tiene que realizar el mismo tipo de deliberación estratégica respecto de la relación medios-fines que en el caso anterior. Pero, además, por otro lado, deberá de tener en cuenta que debe alcanzar ese objetivo dentro del contexto normativo de sus obligaciones militares y dentro del contexto fáctico de las condiciones específicas de la vida castrense. El «soldado de la limpieza» no podrá pretender que, por dedicarse con demasiado ahínco a cumplir con sus responsabilidades particulares, puede dejar de formar filas como cualquier otro soldado, o que puede prescindir de sus

ra lograr tal objetivo. De este modo, la norma no determina ni el conjunto de condiciones en las que debe darse la conducta prescrita ni las acciones o cursos de acción que deben llevarse a cabo», ibídem, p. 10. Así mismo, los autores señalan que las directrices pueden describirse adecuadamente como mandatos de optimización y que en un mismo sujeto pueden concurrir mandatos de optimizar objetivos complementarios o recíprocamente excluyentes. En este sentido, «a diferencia de lo que ocurre con los principios en sentido estricto, en la conducta gobernada por directrices no se trata de determinar la prevalencia de una u otra en relación con un determinado caso, sino de articular políticas capaces de lograr, en el mayor grado posible, la consecución conjunta de todos los objetivos», ibídem. p. 11. Cursivas mías.

${ }^{17}$ Supongamos, para simplificar, que es posible establecer a priori un estándar objetivo de limpieza. 
ejercicios de entrenamiento o del objetivo de llegar a ser un buen soldado en el sentido bélico. Tampoco puede ignorar que en las condiciones de la vida castrense no se puede alcanzar un nivel de limpieza muy alto -menos aún si, por ejemplo, su batallón se encuentra realizando ejercicios de campaña, ni que cuenta con un tiempo limitado para dedicarse a las actividades de aseo. Diremos, pues, que la obligación del soldado de limpiar el cuartel enfrenta limitaciones normativas (otros deberes) y fácticas (condiciones materiales) para realizar su tarea y, por ello, concluiremos que debe procurar que el campamento esté lo más limpio posible dadas esas condiciones.

Me parece claro que cuando consideramos los deberes especiales a la luz de la categoría de las directrices reconstruimos satisfactoriamente las condiciones en las que se formulan juicios de responsabilidad prospectivos; mientras que no es ese el caso cuando tenemos en mente las reglas de fin. Podemos concluir, pues, que las directrices son las normas de mandato que mejor permiten reconstruir la responsabilidad de rol.

\section{Las directrices y las condiciones de justificación de los juicios de responsabilidad}

Ahora bien, que las directrices permitan dar cuenta de los deberes que llamamos «responsabilidades» no supone que este tipo de normas no suscite algunas cuestiones que, desde mi punto de vista, denotan cierto grado de incompatibilidad entre la caracterización de las directrices que antes he expuesto y algunos elementos centrales de la noción de responsabilidad: ¿cómo podemos decir que alguien es responsable -esto es, que tiene una carga- sin establecer las condiciones concretas, el estándar de conducta, a partir del cual consideramos que ha cumplido con ella -esto es, las condiciones en que se descarga-? O dicho en otras palabras, ¿supone la característica sensibilidad a las circunstancias de las directrices que la obligación por ellas prescrita no tiene medida de cumplimiento y que, por tanto, no podemos saber en qué casos alguien ha cumplido con sus responsabilidades? O, en términos del ejemplo del soldado, ¿el hecho de que el soldado tenga objetivos incompatibles y medios limitados para alcanzarlos, es un impedimento para que sus superiores le exijan que dé cuenta de responsabilidades?

Responder a estas preguntas supone ir más allá del ámbito estricto de este trabajo, apartándose de la función a) de la definición, la formulación de juicios de responsabilidad prospectivos, para centrarse en la función b), la formulación de juicios de responsabilidad retrospectivos en los que el término «responsabilidad» opera en el sentido de «sancionabilidad». Requeriría, por decirlo de algún modo, recorrer el camino de vuelta, para mostrar no sólo la manera en la que las directrices permiten dar cuenta de la responsabilidad de rol, sino cómo la noción de responsabilidad permite dar 
cuenta de la dimensión directiva de las directrices. Por su complejidad y extensión esta empresa exige, sin duda, un trabajo autónomo. Sin embargo, para mostrar el potencial explicativo de la vinculación entre la noción de responsabilidad y la categoría normativa de las directrices que he explorado aquí, creo que resulta importante dedicar algunas breves líneas a plantear algunas ideas que, como he apuntado antes, nos conducen a una conclusión paralela o, si se prefiere, complementaria al contenido central del trabajo, a saber: que para ser compatible con la teoría de la responsabilidad, es preciso refinar la caracterización de las directrices como mandatos de optimización de Alexy y de Atienza y Ruiz Manero.

Desde la perspectiva de la teoría de la responsabilidad, responder a las preguntas anteriores requiere detenerse en el sentido de la fórmula «en la mayor medida de lo posible» que cualifica la base normativa de los juicios de responsabilidad como mandatos de optimización. Para ello, es preciso mostrar la forma en la que las directrices constituyen una guía de conducta. $\mathrm{Al}$ respecto, en relación con la noción de responsabilidad hay que tener en cuenta dos cuestiones relacionadas entre sí. Por un lado, aunque existan distintos usos del término «responsabilidad», el concepto central de responsabilidad -hacia el que cabe reconducir, directa o indirectamente, todos esos usos- es el de responsabilidad como «sancionabilidad»; de modo que, para ser coherente con la teoría de la responsabilidad, los juicios de responsabilidad prospectivos que tienen su base normativa en directrices tienen que permitir la formulación justificada de juicios de responsabilidad retrospectivos. Por otro lado, la noción moderna de responsabilidad, que adopta la perspectiva adscriptiva y que reconoce el carácter valorativo de los juicios de responsabilidad retrospectivos, está construida sobre dos pilares que constituyen las principales condiciones de justificación de los juicios de responsabilidad: en primer lugar, la exigencia básica de que el sujeto responsable - esto es, a quien se le adjudica una carga- pueda cumplir con sus responsabilidades - esto es, que pueda descargarse- y, en segundo lugar, el requisito de que dicho cumplimiento tenga en cuenta las circunstancias particulares del sujeto. Estos dos elementos se reflejan en una noción central de la teoría de la responsabilidad: la noción de estándar de conducta. En este sentido, para poder imputar una responsabilidad, para formular un juicio de responsabilidad retrospectivo justificado, se requiere de la aplicación de un estándar de conducta ${ }^{18}$. Tomando esto en cuenta, siempre que hablamos

18 Dentro de la teoría de la responsabilidad, la noción de estándar de conducta se compone, a su vez, de dos partes que constituyen conjuntamente las condiciones de justificación de los juicios de responsabilidad: una parte se refiere a la acción o conducta y la otra a las circunstancias de la misma. Así, para hablar de responsabilidad como sancionabilidad, por ejemplo, en el caso de la responsabilidad por daños o de la responsabilidad penal, se requiere, por 
de «responsabilidad» en un sentido moderno, hablamos de responsabilidad frente a alguien, por un hecho concreto y en unas circunstancias específicas; de modo que se considera que un individuo cumple -o incumple- con sus responsabilidades si sus acciones, en las circunstancias concretas, se ajustan -o no se ajustan- al estándar de conducta en cuestión.

Desde mi punto de vista, el carácter concreto y la dimensión circunstancial de la aplicación de los estándares de conducta resulta iluminadora para esclarecer la virtualidad directiva de las directrices. En esta línea de ideas, para mostrar esta capacidad explicativa conviene recordar que aunque las directrices sean normas que prescriben la obtención de un objetivo, su contenido son conductas y no, naturalmente, estados de cosas. No es posible ordenar estados de cosas sino, en todo caso, las conductas causalmente vinculadas con ellos. En este sentido, hay que tener en mente que aunque la técnica normativa de la formulación de las directrices -y de las reglas de fin- ordene alcanzar un objetivo, se trata de la formulación sintética -o si se prefiere, elíptica- de una norma que ordena realizar ciertas conductas que se caracterizan por tener una relación medio-fin (causal) con el objetivo especificado en la norma. Podemos calificar esas conductas como conductas oportunas.

Así pues, un primer paso para determinar la manera en la que las directrices guían la conducta, consiste en tener en cuenta que mientras que la configuración de las condiciones de aplicación de los principios sólo es sensible a una única dimensión de peso, que se balancea respecto de la misma dimensión de peso de otros principios y que calificamos como jerarquía o prioridad, las directrices, además de esa dimensión de peso, jerarquía o prioridad, tienen una dimensión de oportunidad relativa las posibilidades técnicas para alcanzar el fin social en cuestión. De este modo, para deter-

un lado, que exista una acción u omisión prescrita por una norma de conducta y, por otro, que dicha acción u omisión haya tenido lugar en ciertas circunstancias. Así, sólo se es responsable civil por un daño si, además de demostrar cierta acción o actividad -que, evidentemente, no tiene por qué ser ilícita- se puede demostrar que en el caso concreto existe una relación causal entre la acción o actividad y el daño, y si se puede demostrar la ausencia de ciertos componentes subjetivos relativos a la negligencia o la imprudencia. Por otra parte, sólo hablamos de responsabilidad penal si, además de demostrar la conducta (elemento objetivo), podemos demostrar la ausencia de ciertas condiciones particulares relacionadas, por un lado, con las capacidades de los sujetos (elemento subjetivo) y, por otro, con la relación causal entre el acto antijurídico y sus consecuencias; recogidas ambas bajo la categoría de excusas. En este sentido, a diferencia de la noción de deber, que depende sólo de las condiciones de aplicación de la norma, la noción de responsabilidad es una noción sensible a las circunstancias concretas de la acción relevante. Estos rasgos son los que, por un lado, hacen radicalmente incompatible la noción de responsabilidad con ideas premodernas como la del pecado original y, por otro, generan tensiones entre la teoría estándar de la responsabilidad y conceptos «anómalos» como los de responsabilidad objetiva o responsabilidad colectiva. 
minar la conducta exigida por una directriz, el sujeto debe tener en cuenta las condiciones fácticas en las que tiene lugar la relación medio-fin en cuestión ${ }^{19}$. El primer elemento de los estándares de conducta de las directrices consiste en realizar la(s) conducta(s) oportuna(s).

Ahora bien, ese primer paso tiene que se completado con un segundo, consistente en tener en cuenta una circunstancia universal que juega un papel preeminente entre las condiciones fácticas de la relación medio-fin: la escasez de recursos. En este sentido, el sujeto normativo de una directriz no sólo tiene que tener en cuenta el conjunto de acciones oportunas para dar lugar al objetivo, sino que, además, dentro de ese conjunto tiene que elegir aquella(s) que suponga(n) una relación óptima entre los medios (recursos) y los fines (objetivos).

Así pues, con independencia del criterio de optimalidad que se utilice, en cada momento concreto, dado un determinado conjunto de medios disponibles, el sujeto normativo de una directriz deberá configurar un determinado conjunto de acciones oportunas y eficientes, de modo que dé lugar al objetivo en la mayor medida de lo posible dadas las circunstancias. A esta dimensión de las directrices le podemos llamar dimensión de eficiencia. El segundo elemento de los estándares de conducta de las directrices consiste en realizar la(s) conductas(s) eficiente(s).

A partir de lo anterior, podría afirmarse que mientras que las condiciones de aplicación de los principios en sentido estricto dependen sólo de $\mathrm{ra}$ zones de corrección -i.e., de su encaje respecto del sistema de valores de referencia-, la aplicación de directrices, además de responder a las razones de corrección depende, por un lado, de razones estratégicas -o, si se prefiere, de razones de corrección en sentido técnico- a las que podemos llamar razones de oportunidad y, por otro, de razones referidas a la optimización de los recursos disponibles, a las que podemos llamar razones de eficiencia.

En este sentido, a diferencia de los principios, en los que la ponderación sólo cumple la función de determinar las condiciones de aplicación -i.e. el peso o prevalencia de un principio frente a otro(s) principio(s)-, sin afectar a la guía de conducta determinada de antemano por el propio principio, en el caso de las directrices la ponderación de las razones de oportunidad y eficiencia cumple además la función de configurar el contenido de la guía de conducta. Así, para saber qué conducta(s) debe realizar, el sujeto a quien se le asigna una tarea mediante una directriz debe establecer cuál(es) entre las

${ }^{19}$ En esta dimensión de oportunidad, las directrices no se diferencian de las reglas de fin. La diferencia entre las reglas de fin y las directrices radica, en todo caso, en que las reglas de fin no tienen ni una dimensión de peso ni la dimensión de eficiencia a la que me referiré enseguida. 
conductas oportunas resulta(n) eficiente(s). De este modo, aunque las directrices no establecen a priori una medida de cumplimiento, sí que establecen estándares de conducta respecto de los cuales es posible determinar una medida de cumplimiento específica en cada caso: la realización de la(s) conducta(s) oportunas $y$ eficientes. Consecuentemente, podemos decir que los mandatos de optimización tienen como condición o medida de cumplimiento la utilización eficiente de los recursos disponibles.

En mi opinión, esta conjunción de las dimensiones de oportunidad y eficiencia explica satisfactoriamente tanto la guía de conducta como la medida de cumplimiento de las directrices. Dar cuenta de estos elementos es una condición necesaria para llevar a cabo un análisis satisfactorio de las responsabilidades de rol, del contenido y alcance de la esfera de responsabilidades; pero también para explicar cómo opera este tipo de normas de mandato. Las directrices prescriben realizar las conductas oportunas $y$ eficientes para alcanzar un objetivo. En la medida en la que es posible establecer en cada caso concreto qué conductas tienen esas características, las directrices son susceptibles de ser cumplidas o incumplidas en el mismo sentido en que lo son las reglas y los principios. Desde este punto de vista, la forma prescriptiva de las directrices puede entenderse mejor si tratamos de explicarla, no a partir de la relación contingente entre su contenido y un estado de cosas, sino en virtud de cómo sus condiciones de aplicación afectan la guía de conducta. Esto es, si analizamos su funcionamiento, por un lado, desde la perspectiva de la justificación de juicios de responsabilidad y, por otro, a partir de su relación con el tipo de razones que tienen que tenerse cuenta en la deliberación respecto del curso de acción efectivamente prescrito -i.e. de la determinación de estándares de conducta-. Dicho en otros términos, con independencia de que el estado de cosas en cuestión pueda alcanzarse plenamente o sólo de manera gradual o marginal ${ }^{20}$, hablamos de mandatos de optimización si, y sólo si, el objetivo en cuestión se establece de modo que para su obtención deba tenerse en cuenta la optimización de los recursos disponibles en las circunstancias concretas de la acción. Así, en el ejemplo del soldado, aunque la limpieza del campamento sea un resultado que admite distintos grados de consecución y, en principio, no podamos establecer cuándo consideraremos que el campamento esté limpio, no es ésa la razón por la que decimos que existe un mandato de optimización. El soldado es sujeto de un mandato de optimización porque, para alcanzar cualquier nivel

${ }^{20}$ Se trataría, en el primer caso, de lo que Robert E. Goodin ha llamado «responsabilidades de objetivo determinado» (fixed-target responsibility) y, en el segundo, de lo que ha denominado «responsabilidades de objetivo recesivo» (receding-target responsibility). Cfr. Goodin, Robert E., Utilitarianism as a Public Philosophy, Cambridge University Press, 1995, p. 86. 
de limpieza del campamento -con independencia de cuál sea éste-, tiene que utilizar de la mejor manera posible los recursos de los que dispone. Así, como he señalado antes, el soldado tiene, por ejemplo, que asignar de manera eficiente el escaso tiempo que las demás tareas que conlleva su oficio de soldado le dejen libre.

La articulación de las razones de oportunidad y de eficiencia, y los juicios de prudencia que éstas implican, constituyen el problema central de la integración de la esfera de responsabilidades. La especificación de los criterios mediante los cuales imputamos las consecuencias del cumplimiento o incumplimiento de los deberes dentro de tal esfera está en el núcleo de una teoría de la responsabilidad completa y bien estructurada que, desde luego, no es posible abordar en un trabajo como éste. Sin embargo, creo que se puede concluir provisionalmente que no sólo la categoría de las directrices ayuda a esclarecer la responsabilidad de rol, sino que la teoría de la responsabilidad constituye un buen punto de partida para entender mejor cómo operan los mandatos contenidos en las directrices. De ello, me ocuparé en otro momento. 
$\triangle \quad$ DOXA 24 (2001) 